\title{
Article \\ Self-Reported Walking Difficulty Associated with Stiff-Knee Gait in Japanese Patients with Knee Osteoarthritis: A Preliminary Cross-Sectional Study
}

\author{
Haruki Toda $^{1, *(\mathbb{D})}$, Tsubasa Maruyama ${ }^{1}$, Koji Fujita ${ }^{2}\left(\mathbb{D}\right.$, Yuki Yamauchi ${ }^{3}$ and Mitsunori Tada ${ }^{1}(\mathbb{D})$ \\ 1 Digital Human Research Team, Artificial Intelligence Research Center, National Institute of Advanced \\ Industrial Science and Technology, Tokyo 135-0064, Japan; tbs-maruyama@aist.go.jp (T.M.); \\ m.tada@aist.go.jp (M.T.) \\ 2 Department of Functional Joint Anatomy, Graduate School of Medical and Dental Sciences, Tokyo Medical \\ and Dental University, Tokyo 113-8519, Japan; fujiorth@tmd.ac.jp \\ 3 Department of Orthopaedic Surgery, Doujin Hospital, Urasoe 901-2133, Japan; yamauchi.orth@tmd.ac.jp \\ * Correspondence: haruki-toda@aist.go.jp; Tel.: +81-3-3599-8201
}

\section{check for} updates

Citation: Toda, H.; Maruyama, T.; Fujita, K.; Yamauchi, Y.; Tada, M. Self-Reported Walking Difficulty Associated with Stiff-Knee Gait in Japanese Patients with Knee Osteoarthritis: A Preliminary Cross-Sectional Study. Healthcare 2021, 9, 1308. https://doi.org/10.3390/ healthcare 9101308

Academic Editors: Tadashi Ito and Jose M. Moran

Received: 29 July 2021

Accepted: 27 September 2021

Published: 30 September 2021

Publisher's Note: MDPI stays neutral with regard to jurisdictional claims in published maps and institutional affiliations.

Copyright: (c) 2021 by the authors. Licensee MDPI, Basel, Switzerland. This article is an open access article distributed under the terms and conditions of the Creative Commons Attribution (CC BY) license (https:// creativecommons.org/licenses/by/ $4.0 /)$.

\begin{abstract}
Background: Individuals with knee osteoarthritis are restricted in their daily activity because of walking difficulty. The purpose of this investigation was to examine the association between self-reported walking difficulty and knee flexion excursion during gait in Japanese patients with knee osteoarthritis. Methods: Twenty-eight patients with knee osteoarthritis participated in this study. Knee flexion excursions in loading response and swing during gait were measured through an inertial measurement unit-based motion capture system. The walking difficulty was assessed by a subitem in the Japanese Knee Osteoarthritis Measure. Pain intensity was assessed by a visual analog scale. Characteristics and gait variables were compared between groups that were determined a priori using the results of the walking difficulty assessment. The relationship between knee flexion excursion during gait and walking difficulty were analyzed using logistic regression. Results: The participants with walking difficulty had significantly small knee flexion excursion in both loading response and swing with large pain. After controlling the effect of pain, only knee flexion excursion in the swing was significantly related to the walking difficulty. Conclusions: This study suggested that the knee flexion excursion in swing during gait is helpful for understanding the walking difficulty experienced in Japanese patients with knee osteoarthritis.
\end{abstract}

Keywords: gait; inertial measurement unit; knee flexion excursion; knee osteoarthritis; motion capture; swing; walking difficulty

\section{Introduction}

Knee osteoarthritis (OA) is a major musculoskeletal problem among the elderly. In Japan, the prevalence of radiographic knee OA has been reported to be $48.2 \%$ in males and $71.9 \%$ in females aged 70-79 years [1]. Knee OA limits the activities of daily living [2]. Furthermore, knee OA is a potentially modifiable risk factor for serious cardiovascular diseases and death [3]. To reduce the risk of developing functional limitations, people with knee OA are recommended to walk over 6000 steps/day [4]. In addition, walking for at least $30 \mathrm{~min}$ is considered a moderate aerobic exercise that can help to prevent the progression of knee OA [5]. Although walking is a simple motion that can be performed in daily living, many individuals with knee OA avoid walking during their daily activities due to knee pain and stiffness [6,7]. Therefore, to promote walking, the perceived difficulty among patients with knee OA should be minimized.

Walking difficulty in Japanese patients with knee OA should be assessed according to their lifestyle and sense of values. Previous studies reported by $\mathrm{Na}$ et al. [8,9] examined walking difficulty in patients with knee OA using a subitem of the Knee Outcome Survey 
(KOS). However, the results of this evaluation may be influenced by differences in crosscultural backgrounds if adapted to knee OA among Japanese people. Therefore, Akai et al., [10] developed the Japanese Knee Osteoarthritis Measure (JKOM) based on the International Classification of Functioning, Disability, and Health. JKOM subitems also have questions regarding walking difficulty on flat surfaces.

Individuals with knee OA exhibit a stiff-knee gait pattern characterized by small knee flexion excursion during the loading response and swing phase [11,12]. Previous studies have reported the association of knee kinematics and kinetics during the early stance of walking with the self-reported walking difficulty evaluated by KOS among individuals with knee OA [8,9]. However, to our knowledge, no study has examined the relationship between self-reported walking difficulty evaluated by JKOM and knee flexion excursion in both loading response and swing among Japanese patients with knee OA.

The stiff-knee gait pattern is caused by abnormal muscle activation around the knee [13] and increases mechanical stress in the knee [14]. Knee flexion excursion in the loading response contributes to attenuating the impact load on the articular cartilage by weight acceptance. Additionally, in the swing phase, the knee flexion motion to ensure toe clearance is required to avoid the risk of falling, especially on an uneven surface in the outdoor environment. Moreover, inadequate knee flexion in the swing induces energyinefficient compensatory movements [15]. Stiff-knee gait patterns may hence contribute to the self-reported walking difficulty among individuals with knee OA.

This study aimed to examine the relationship between self-reported walking difficulty and knee flexion excursion in loading response and swing among Japanese patients with knee OA. We hypothesize that participants with small knee flexion excursions during gait show a higher possibility of experiencing walking difficulties in their daily living than those with large knee flexion excursions.

\section{Materials and Methods}

\subsection{Participants}

Individuals with knee OA participated in this study. All participants were diagnosed with tibiofemoral knee OA through an evaluation using weight-bearing anteroposterior radiographs with the Kellgren-Lawrence (K-L) classification of grade 1 or higher in addition to clinical symptoms. The participants could walk independently without any assistive devices. If the participant had knee OA on both lower limbs, we selected the more symptomatic side. The exclusion criteria included neurologic disorders, trauma, and a history of knee surgery.

\subsection{Clinical Evaluation}

Knee OA severity was assessed using the K-L grade [16]. K-L grade and femorotibial angle (FTA) were evaluated using weight-bearing anteroposterior radiographs.

The participants completed a self-administered questionnaire using the visual analog scale (VAS) as well as the JKOM [10]. VAS was used to evaluate the degree of pain in the knee during walking on a scale of $0-100 \mathrm{~mm}$. Walking difficulty was assessed using the JKOM subitem regarding walking on a flat surface: "How difficult can you walk on a flat surface without taking a rest? $(0=$ over $30 \mathrm{~min}, 1=$ about $15 \mathrm{~min}, 2=$ around your house, $3=$ only into your house, $4=$ almost not)". Participants who scored 0 were assigned into the non-difficulty (non-Diff) group, whereas participants who scored $\geq 1$ were assigned into the difficulty (Diff) group.

\subsection{Range of Motion}

A plastic goniometer (OG wellness Co., Ltd, Okayama, Japan) was used to measure the passive knee extension range of motion $(\mathrm{RoM})$ in $5^{\circ}$ increments. The participants were examined in the supine position on an examination table. The goniometer was aligned over the sagittal axis of the thigh and shank. 


\subsection{Muscle Strength}

A hand-held dynamometer (HHD) ( $\mu$ Tas F-1; Anima Corp., Tokyo, Japan) was used to measure maximum isometric knee extension and flexion muscle strength. The participants were examined in a sitting position on an examination table with the hip and knee joints at $90^{\circ}$ flexion, the lower leg perpendicular to the floor and the feet not touching the floor, according to a previous study [17]. The HHD sensor was attached to in front of and behind the distal end of the shank during extension and flexion, respectively. Measurements were taken for $5 \mathrm{~s}$ each after the practice trials. The measured forces were normalized by shank length and body weight $(\mathrm{Nm} / \mathrm{kg})$.

\subsection{Gait Measurement}

Whole-body motion during walking was measured using an inertial measurement unit (IMU)-based motion capture system. This system was developed by Maruyama et al. [17] and validated in our previous studies [18,19]. Thirteen IMUs (MTw; Xsens Technologies Inc., Enschede, The Netherlands) were attached to each body segment of the participants in accordance with our previous study [20]. The participants were allowed to use their shoes during walking to ensure high fidelity in measuring the knee's natural function [10]. A reference pose was taken prior to the walking trials for calibration of the IMU orientation to the corresponding body segment. Afterwards, the participants walked approximately $12 \mathrm{~m}$ in a straight walkway at the hospital with a self-selected comfortable walking speed.

\subsection{Data Analysis}

A posture-reconstruction plugin running on DhaibaWorks, a self-developed motion analysis software described previously [21], was used for the knee joint angle calculation at $60 \mathrm{~Hz}$. This plugin reconstructed the lower limb motion by combining the orientation of IMUs attached to body segments and the individual body model through a link structure. The dimensions of the model have been estimated statistically from the participants' body height and weight based on the Japanese body dimensions database [20]. In addition, we reflected participants' FTA and passive knee extension RoM in the body models.

A Butterworth low-pass filter at $6 \mathrm{~Hz}$ was applied to the kinematic data. Data for five gait cycles during steady-state walking were extracted. The knee flexion excursions in the loading response and swing were calculated from the displacements between the maximum extension angle around the initial contact and the maximum flexion angle in the loading response, and the maximum extension angle in the terminal stance and the maximum flexion angle in the swing phase, respectively (Figure 1). The stride length was calculated as the distance between the heel points projected on the sagittal plane. The walking speed was calculated from the stride length and the duration of one gait cycle time.

\subsection{Statistical Analysis}

We used the Mann-Whitney U-test to examine continuous variables and the chi-square test to examine categorical variables between the non-Diff and Diff groups.

Logistic regression analysis was conducted with walking difficulty (non-difficulty or difficulty) as the dependent variable. We created two logistic regression models to determine whether knee flexion excursion in loading response (Model 1) and swing (Model 2) affects walking difficulty. In addition, the variables that differed between the non-Diff and Diff groups were entered into each model as an adjustment variable using forced entry.

The significance level was set at 0.05 . All data were analyzed using the SPSS statistical software version 25.0 (IBM, Armonk, NY, USA). 


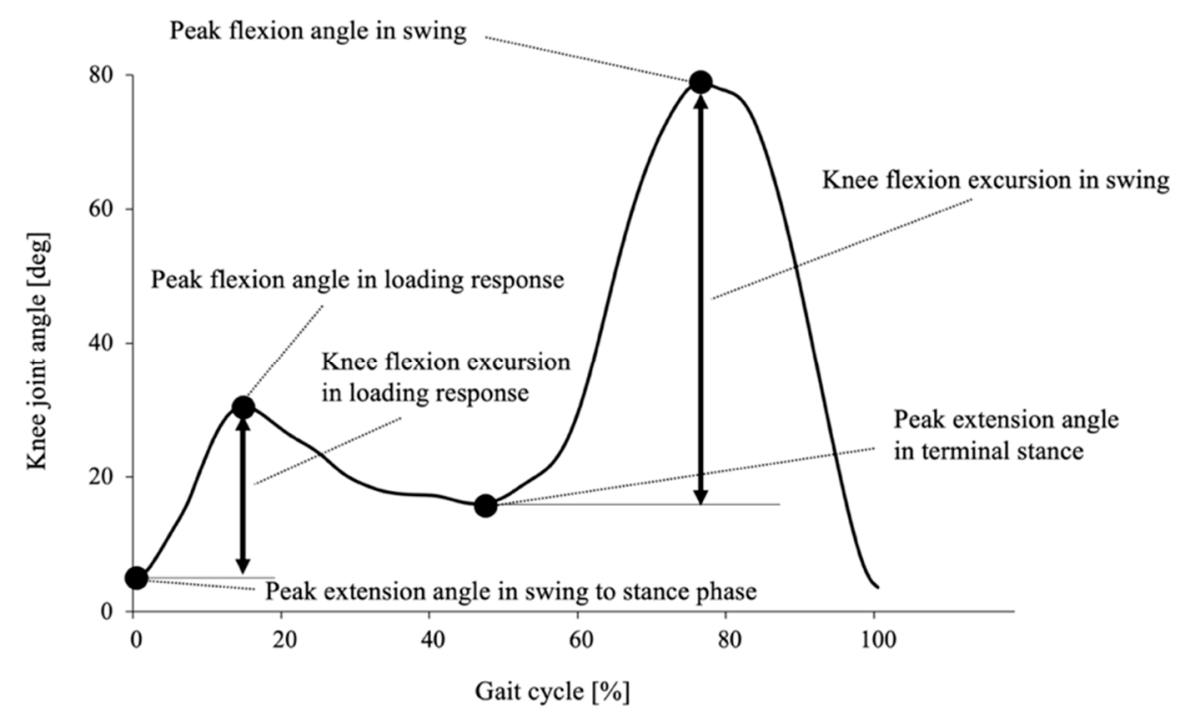

Figure 1. Typical knee flexion angle of a subject in one gait cycle. The knee flexion excursions were calculated from the amplitude of displacement from the peak extension angle to the peak flexion angle during the loading response and swing phase.

\section{Results}

Twenty-eight patients with knee OA participated in this study. Nineteen participants were classified into the non-Diff group, whereas nine participants were classified into the Diff group (walking on a flat surface; JKOM scores were as follows: score of 1 = five participants, score of $2=$ one participants, score of $3=$ three participants). Table 1 shows the characteristics of the participants in each group.

Table 1. Characteristics and outcome measures of the groups with walking difficulty (Diff) and without walking difficulty (non-Diff).

\begin{tabular}{cccc}
\hline Characteristics & Non-Diff Group $(\boldsymbol{n = 1 9 )}$ & Diff Group $(\boldsymbol{n}=\mathbf{9 )}$ & $\boldsymbol{p}$-Value \\
\hline Age (years) & $72.8 \pm 10.1$ & $72.8 \pm 11.5$ & 0.847 \\
Sex $(n)$ & & & 0.483 \\
Male & $4(21.1 \%)$ & $3(33.3 \%)$ & \\
Female & $15(78.9 \%)$ & $6(66.7 \%)$ & 0.847 \\
Height $(\mathrm{m})$ & $1.53 \pm 0.09$ & $1.54 \pm 0.11$ & 0.885 \\
Weight $(\mathrm{kg})$ & $58.7 \pm 9.7$ & $58.8 \pm 8.6$ & 0.962 \\
BMI $\left(\mathrm{kg} / \mathrm{m}^{2}\right)$ & $24.8 \pm 3.1$ & $24.7 \pm 3.0$ & 0.259 \\
K-L grade $(n)$ & & & \\
Grade I & $4(21.1 \%)$ & $1(11.1 \%)$ & \\
Grade II & $7(36.8 \%)$ & $1(11.1 \%)$ & \\
Grade III & $4(21.1 \%)$ & $5(55.6 \%)$ & \\
Grade IV & $4(21.1 \%)$ & $2(22.2 \%)$ & 0.142 \\
Muscle strength & & & 0.085 \\
(Nm/kg) & & & \\
Flexion & $0.66 \pm 0.18$ & $0.54 \pm 0.11$ & 0.156 \\
Extension & $1.09 \pm 0.28$ & $0.90 \pm 0.24$ & \\
VAS (mm) & $37.0 \pm 31.8$ & $58.7 \pm 27.6$ & \\
Walking speed (m/s) & $1.18 \pm 0.32$ & $0.98 \pm 0.24$ &
\end{tabular}

Value: Mean \pm standard deviation. ${ }^{*} p<0.05$. BMI, body mass index; K-L, Kellgren-Lawrence grade; VAS, visual analog scale.

There were no significant differences in age, sex, height, weight, body mass index, $\mathrm{K}-\mathrm{L}$ grade, muscle strength, and walking speed between the non-Diff and Diff groups. In contrast, the Diff group had significantly smaller knee flexion excursions in both loading response and swing, with larger VAS score during walking than the non-Diff group (Table 1 and Figure 2). 

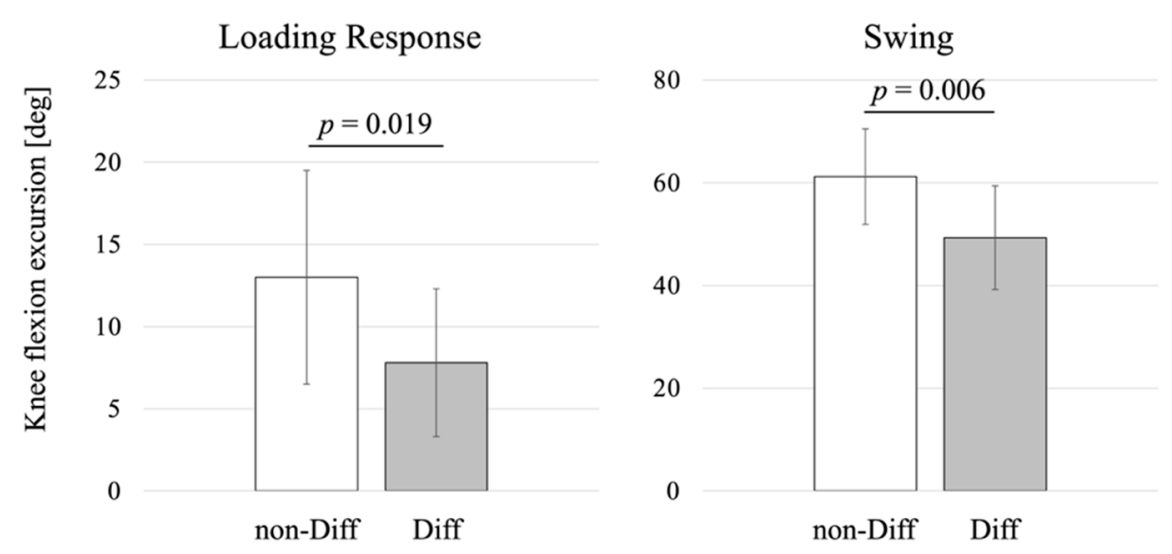

Figure 2. Comparison of knee flexion excursions in loading response and swing between the groups with walking difficulty (Diff) and without walking difficulty (non-Diff).

Table 2 shows the results of the logistic regression analysis. Because the VAS scores were significantly different between the groups, this variable was entered into the logistic regression models, with the knee flexion excursions in loading response and swing. As a result, the knee flexion excursion in the swing was observed to be significantly related to walking difficulty. In contrast, the knee flexion excursion in the loading response was not statistically significant after controlling for the effect of the VAS walking.

Table 2. Results of the logistic regression analysis between the groups with walking difficulty (Diff) and without walking difficulty (non-Diff).

\begin{tabular}{|c|c|c|c|c|c|}
\hline & \multirow{2}{*}{ Variables } & \multirow{2}{*}{$\mathrm{OR}^{1}$} & \multicolumn{2}{|c|}{$95 \% \mathrm{CI}^{2}$} & \multirow{2}{*}{$p$-Value } \\
\hline & & & Lower & Upper & \\
\hline \multirow[t]{2}{*}{ Model 1} & $\begin{array}{l}\text { Knee flexion excursion in } \\
\text { loading response }\end{array}$ & 0.835 & 0.690 & 1.011 & 0.064 \\
\hline & VAS $^{3}$ & 1.049 & 1.005 & 1.085 & 0.027 * \\
\hline \multirow[t]{2}{*}{ Model 2} & Knee flexion excursion in swing & 0.730 & 0.560 & 0.952 & 0.020 * \\
\hline & VAS $^{3}$ & 1.086 & 1.010 & 1.154 & $0.025 *$ \\
\hline
\end{tabular}

${ }^{*} p<0.05 .{ }^{1} \mathrm{OR}$, odds ratio; ${ }^{2} \mathrm{CI}$, confidence interval; ${ }^{3} \mathrm{VAS}$, visual analog scale.

\section{Discussion}

This study examined whether the stiff-knee gait pattern of individuals with knee OA was related to self-reported walking difficulty in daily living, and we found that the knee flexion excursion in the swing was associated with difficulty in walking on a flat surface (Figure 3), which partially supports our hypothesis.

Knee flexion motion in the swing was significantly related to the difficulty of walking on a flat surface, despite controlling for the factor of knee pain. The present study showed that the individuals with small knee flexion excursion in the swing were likely to experience walking difficulty in daily living, which could imply that this motion is helpful for understanding the restrictions in walking duration and environment regardless of the knee pain. Generally, a small knee flexion motion in the swing is caused by the overactivity of the quadriceps [22]. This motion controls the toe clearance [23]. Individuals with knee OA who had a small knee flexion excursion in the swing have a large stumbling risk because of the difficulty in adapting to changes in the environment [24]. Avoiding stumbling, these individuals adopted to the compensatory trunk and lower limb movements [25]. In addition, the overactivity and compensatory movements caused by stiff-knee gait patterns lead to higher energy costs [26]. A previous study reported that a small range of knee flexion-extension in a gait cycle was correlated with a short six-minute walk distance in individuals with knee OA [27]. Decreasing the knee flexion in the swing might also reduce walking activity in terms of energy costs and the risk of stumbling. This suggests that 
interventions for the inhibition of the activity of the quadriceps in the terminal stance and swing assistance using a wearable pneumatic artificial muscle [28] might effectively increase the walking activity by minimizing the walking difficulty in individuals with knee $\mathrm{OA}$, and further research is warranted.

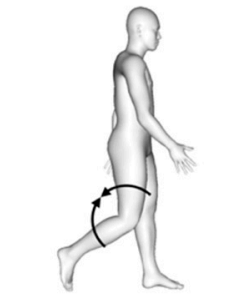

Knee flexion in swing
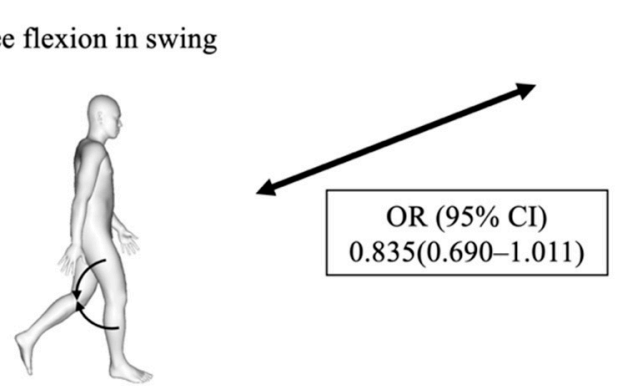

Knee flexion in loading response

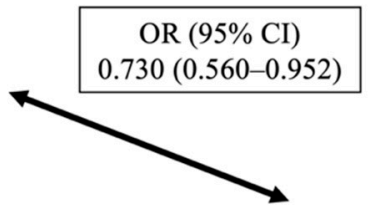

Walking difficulty

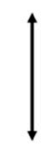

Knee pain

Figure 3. Graphical abstract of this study. Self-reported walking difficulty in Japanese patients with knee osteoarthritis was associated with the knee flexion excursion in the swing. The knee flexion excursion in the loading response is not related to the walking difficulty after controlling for the factor of knee pain. Odds ratios (OR) with their $95 \%$ confidence intervals (CI) are provided.

The Diff group had a small knee flexion excursion during the loading response. In contrast, a previous study by Na et al. [8] reported that the knee flexion excursion in the loading response did not differ between patients with and without walking difficulty, which is inconsistent with our results. There may be a few reasons for this difference. The previous study evaluated the walking difficulty using a KOS, which asked participants to feel the difficulty while walking. In contrast, our study employed the JKOM, which asked about the participants' walking activity in daily life, such as the duration and environment. The contents of the respective questions may have affected the participant grouping. In addition, although $\mathrm{Na}$ et al. [8] controlled the subjects' walking speed, our participants walked at a comfortable speed. The different experimental designs may have also affected the results. Nevertheless, our study results suggest that the relationship between the walking difficulty and the knee flexion excursion in the loading response was small, based on logistic regression analysis.

The participants in the Diff group had significantly higher VAS score than those in the non-Diff group. Pain and its related fear are characterized by escape and avoidance behaviors, and avoidance of daily activities results in functional disability [29]. The JKOM assessed the walking difficulty by asking about the walking duration and environment in daily living. Since participants in the Diff group experienced considerable pain, their walking duration and environment could be restricted by the pain-related avoidance of daily activities. Moreover, knee pain induces small knee excursions to reduce the external force generated in the knee joint [11]. Knee pain affected the relationship between the walking difficulty and small knee excursions in the loading response as a confounding variable. Although the participants with a small knee flexion excursion in the loading response felt that walking difficulty, the effect on walking difficulty was small when controlling for the factor of knee pain.

This study had some limitations. First, walking difficulty was evaluated using a selfadministrated questionnaire. Patient-reported outcome measures can be easily evaluated 
in a clinical setting. On the other hand, walking area and time and the frequency of use of a cane can be quantitatively measured using a wearable sensor, that is, an IMU and a pedometer. Further studies are needed to examine the relationship between walking activity in daily living as measured by wearable sensors and joint kinematics during walking. Second, this study employed a cross-sectional design. Therefore, we could not identify a causal relationship between walking difficulty and knee flexion excursion based on the results. Third, this study is a preliminary study with a small sample size. To generalize the results of this study, a homogenized group with a larger sample size is necessary. Finally, in this study, no evaluations were performed using the Western Ontario and McMaster Universities Osteoarthritis Index and Knee Outcome Survey, which are international knee OA scales. Thus, we cannot compare the results of this study to studies that used the international scales.

\section{Conclusions}

This study revealed that individuals with knee OA exhibit the stiff-knee gait pattern, wherein the small knee flexion excursion in the swing was associated with the self-reported walking difficulty in daily living. The results suggest that to increase their walking activity, interventions to increase knee flexion excursion in the swing should be taken into consideration.

Author Contributions: Conceptualization, H.T.; methodology, H.T., K.F. and Y.Y.; software, M.T. and T.M.; validation, H.T. and T.M.; formal analysis, H.T.; investigation, H.T., K.F. and Y.Y.; resources, M.T.; data curation, H.T., K.F. and Y.Y.; writing-original draft preparation, H.T.; writing-review and editing, T.M., K.F., Y.Y. and M.T.; visualization, H.T.; supervision, M.T.; project administration, K.F.; funding acquisition, M.T. All authors have read and agreed to the published version of the manuscript.

Funding: This work was supported by JST AIP-PRISM [grant number JPMJCR18Y2].

Institutional Review Board Statement: The study was conducted according to the guidelines of the Declaration of Helsinki and approved by the local institutional review board of Tokyo Medical and Dental University (M2018-123).

Informed Consent Statement: Informed consent was obtained from all subjects involved in the study. Data Availability Statement: Data sharing not applicable.

Acknowledgments: The authors thank the staff of the Tokyo Medical and Dental University Hospital and the Doujin Hospital for the assistance with data collection.

Conflicts of Interest: The authors declare no conflict of interest.

\section{References}

1. Yoshimura, N.; Muraki, S.; Oka, H.; Mabuchi, A.; En-Yo, Y.; Yoshida, M.; Saika, A.; Yoshida, H.; Suzuki, T.; Yamamoto, S.; et al. Prevalence of knee osteoarthritis, lumbar spondylosis, and osteoporosis in Japanese men and women: The research on osteoarthritis/osteoporosis against disability study. J. Bone Miner. Metab. 2009, 27, 620-628. [CrossRef] [PubMed]

2. Vongsirinavarat, M.; Nilmart, P.; Somprasong, S.; Apinonkul, B. Identification of knee osteoarthritis disability phenotypes regarding activity limitation: A cluster analysis. BMC Musculoskelet. Disord. 2020, 21, 237. [CrossRef]

3. Hawker, G.A.; Croxford, R.; Bierman, A.S.; Harvey, P.J.; Ravi, B.; Stanaitis, I.; Lipscombe, L.L. All-Cause Mortality and Serious Cardiovascular Events in People with Hip and Knee Osteoarthritis: A Population Based Cohort Study. PLoS ONE 2014, 9, e91286. [CrossRef] [PubMed]

4. White, D.K.; Tudor-Locke, C.; Zhang, Y.; Fielding, R.; LaValley, M.; Felson, D.; Gross, K.D.; Nevitt, M.C.; Lewis, C.E.; Torner, J.; et al. Daily Walking and the Risk of Incident Functional Limitation in Knee Osteoarthritis: An Observational Study. Arthritis Rheum. 2014, 66, 1328-1336. [CrossRef] [PubMed]

5. Loew, L.; Brosseau, L.; Wells, G.A.; Tugwell, P.; Kenny, G.P.; Reid, R.; Maetzel, A.; Huijbregts, M.; McCullough, C.; De Angelis, G.; et al. Ottawa Panel Evidence-Based Clinical Practice Guidelines for Aerobic Walking Programs in the Management of Osteoarthritis. Arch. Phys. Med. Rehabil. 2012, 93, 1269-1285. [CrossRef]

6. Van Dijk, G.M.; Veenhof, C.; Schellevis, F.; Hulsmans, H.; Bakker, J.P.; Arwert, H.; Dekker, J.H.; Lankhorst, G.J.; Dekker, J. Comorbidity, limitations in activities and pain in patients with osteoarthritis of the hip or knee. BMC Musculoskelet. Disord. 2008, 9, 1-10. [CrossRef] 
7. Quicke, J.; Foster, N.; Thomas, M.; Holden, M. Is long-term physical activity safe for older adults with knee pain? A systematic review. Osteoarthr. Cartil. 2015, 23, 1445-1456. [CrossRef]

8. Na, A.; Piva, S.R.; Buchanan, T.S. Influences of knee osteoarthritis and walking difficulty on knee kinematics and kinetics. Gait Posture 2018, 61, 439-444. [CrossRef]

9. Na, A.; Buchanan, T.S. Self-reported walking difficulty and knee osteoarthritis influences limb dynamics and muscle co-contraction during gait. Hum. Mov. Sci. 2018, 64, 409-419. [CrossRef]

10. Akai, M.; Doi, T.; Fujino, K.; Iwaya, T.; Kurosawa, H.; Nasu, T. An outcome measure for Japanese people with knee osteoarthritis. J. Rheumatol. 2005, 32, 1524-1532.

11. Favre, J.; Erhart-Hledik, J.; Andriacchi, T. Age-related differences in sagittal-plane knee function at heel-strike of walking are increased in osteoarthritic patients. Osteoarthr. Cartil. 2014, 22, 464-471. [CrossRef]

12. McCarthy, I.; Hodgins, D.; Mor, A.; Elbaz, A.; Segal, G. Analysis of knee flexion characteristics and how they alter with the onset of knee osteoarthritis: A case control study. BMC Musculoskelet. Disord. 2013, 14, 169. [CrossRef] [PubMed]

13. Smith, S.L.; Allan, R.; Marreiros, S.P.; Woodburn, J.; Steultjens, M.P.M. Muscle Co-Activation Across Activities of Daily Living in Individuals with Knee Osteoarthritis. Arthritis Rheum. 2019, 71, 651-660. [CrossRef] [PubMed]

14. Trepczynski, A.; Kutzner, I.; Schwachmeyer, V.; Heller, M.O.; Pfitzner, T.; Duda, G.N. Impact of antagonistic muscle co-contraction on in vivo knee contact forces. J. Neuroeng. Rehabil. 2018, 15, 101. [CrossRef] [PubMed]

15. Fox, M.D.; Reinbolt, J.A.; Ounpuu, S.; Delp, S.L. Mechanisms of improved knee flexion after rectus femoris transfer surgery. J. Biomech. 2009, 42, 614-619. [CrossRef]

16. Kellgren, J.H.; Lawrence, J.S. Radiological Assessment of Osteo-Arthrosis. Ann. Rheum. Dis. 1957, 16, 494-502. [CrossRef] [PubMed]

17. Wang, C.-Y.; Olson, S.L.; Protas, E.J. Test-retest strength reliability: Hand-held dynamometry in community-dwelling elderly fallers. Arch. Phys. Med. Rehabil. 2002, 83, 811-815. [CrossRef]

18. Maruyama, T.; Toda, H.; Ishii, W.; Tada, M. Inertial Measurement Unit to Segment Calibration Based on Physically Constrained Pose Generation. SICE J. Control Meas. Syst. Integr. 2020, 13, 122-130. [CrossRef]

19. Toda, H.; Maruyama, T.; Tada, M. Indoor vs. Outdoor Walking: Does It Make Any Difference in Joint Angle Depending on Road Surface? Front. Sports Act. Living 2020, 2, 119. [CrossRef]

20. Toda, H.; Maruyama, T.; Fujita, K.; Yamauchi, Y.; Tada, M. Self-Perception of the Knee Is Associated with Joint Motion during the Loading Response in Individuals with Knee Osteoarthritis: A Pilot Cross-Sectional Study. Sensors 2021, 21, 4009. [CrossRef]

21. Endo, Y.; Tada, M.; Mochimaru, M. Dhaiba: Development of virtual ergonomic assessment system with human models. In Proceedings of the 3rd International Digital Human Symposium, Tokyo, Japan, 20-22 May 2014.

22. Goldberg, S.R.; Anderson, F.C.; Pandy, M.; Delp, S.L. Muscles that influence knee flexion velocity in double support: Implications for stiff-knee gait. J. Biomech. 2004, 37, 1189-1196. [CrossRef] [PubMed]

23. Moosabhoy, M.A.; Gard, S. Methodology for determining the sensitivity of swing leg toe clearance and leg length to swing leg joint angles during gait. Gait Posture 2006, 24, 493-501. [CrossRef] [PubMed]

24. Arnold, C.M.; Gyurcsik, N.C. Risk Factors for Falls in Older Adults with Lower Extremity Arthritis: A Conceptual Framework of Current Knowledge and Future Directions. Physiother. Can. 2012, 64, 302-314. [CrossRef] [PubMed]

25. Kerrigan, D.C.; Frates, E.P.; Rogan, S.; Riley, P.O. Hip hiking and circumduction: Quantitative definitions. Am. J. Phys. Med. Rehabil. 2000, 79, 247-252. [CrossRef]

26. Lewek, M.D.; Osborn, A.J.; Wutzke, C. The Influence of Mechanically and Physiologically Imposed Stiff-Knee Gait Patterns on the Energy Cost of Walking. Arch. Phys. Med. Rehabil. 2012, 93, 123-128. [CrossRef]

27. Maly, M.R.; Costigan, P.A.; Olney, S.J. Role of knee kinematics and kinetics on performance and disability in people with medial compartment knee osteoarthritis. Clin. Biomech. 2006, 21, 1051-1059. [CrossRef]

28. Toda, H.; Maruyama, T.; Kurita, Y.; Tada, M. Individual Adjustment of Contraction Parameters for Effective Swing Assist Using a Pneumatic Artificial Muscle in the Elderly. Appl. Sci. 2021, 11, 4308. [CrossRef]

29. Vlaeyen, J.W.; Linton, S.J. Fear-avoidance and its consequences in chronic musculoskeletal pain: A state of the art. Pain 2000, 85, 317-332. [CrossRef] 